\title{
Der Schutz der Gesundheit und der des Arztgeheimnisses gehen Hand in Hand
}

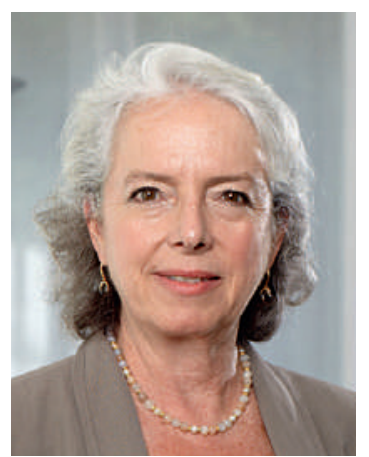

Der Sommer bietet eine gute Gelegenheit, um mit einem gewissen Abstand über die Fragilität unseres Gesundheitssystems nachzudenken. Fragt man die Schweizer Bevölkerung, so ist sie einfach nur stolz auf unser Gesundheitswesen - und die Zahlen scheinen ihr recht zu geben: Im Vergleich $\mathrm{zu}$ anderen entwickelten Ländern ist unsere Lebenserwartung ausgezeichnet (Männer 79,9, Frauen 84,6 im Jahr 2009, wobei das Durchschnittsalter insgesamt von 80,6 im Jahr 2003 auf 82,3 im Jahr 2009 angestiegen ist). Aber wir können uns nicht auf unseren Lorbeeren ausruhen: Der Einfluss politischer und gesellschaftlicher Entscheidungen auf die Gesundheit eines Volkes ist enorm, wie der Bericht von Steven H. Woolf und Laudan Aron über die Gesundheit in den USA [1] auf besonders beunruhigende Weise aufzeigt. Denn trotz des hohen Lebensstandards und Gesundheitskosten, die zu den höchsten der Welt gehören, ist der Gesundheitszustand der amerikanischen Bevölkerung, verglichen mit 17 anderen entwickelten Ländern, darunter auch die Schweiz, der schlechteste - dies unabhängig vom Alter und der sozialen Herkunft.

\section{Der Aufbau eines guten therapeutischen Netzwerks setzt voraus, dass Ärztinnen und Ärzte auf ihren Erfahrungen aufbauen können.}

«Gesundheit resultiert nicht aus einzelnen Faktoren, sondern aus vielfältigen Interaktionen und Synergien», schreibt B. Kiefer in seinem Kommentar [2]. Der niederschwellige Zugang zu Gesundheitsleistungen ist dabei wesentlich, und dafür ist wiederum das Arztgeheimnis Grundvoraussetzung. Dieses schützt das Vertrauensverhältnis, die sorgfältige Ausübung der medizinischen Berufe und dient somit auch der öffentlichen Gesundheit. Um das öffentliche Vertrauen in den Arztberuf zu sichern, muss der Arzt sogar dann das Geheimnis bewahren können, wenn ihn der Patient von der Schweigepflicht entbunden hat. Die Bewahrung des Arztgeheimnisses hat ihren Preis, und man muss sich dafür engagieren.

Auch im europäischen Recht [3] geniesst der Schutz persönlicher Daten Priorität. Daten dürfen nicht mehr als erforderlich gesammelt oder weitergegeben werden («Datensparsamkeit»). Der Schutz der Privatsphäre muss bei jedem Projekt von Beginn an berücksichtigt werden. Die Kontrolle über die eigenen Daten muss selbstverständlich die betroffene Person selbst haben: Sie hat ein Recht auf Information, Korrektur und Löschung der Daten, ja sogar ein «Recht auf Vergessen». Die Regulierungsbehörden sind verpflichtet einzugreifen, und ihre Unabhängigkeit muss gewährleistet sein.
Dies alles steht im Gegensatz zu den Strömungen einer transparenzgläubigen Gesellschaft. Aber das Arztgeheimnis kann auch beunruhigen und die Befürchtung wecken, die Ärztinnen und Ärzte wollten damit eigene Versäumnisse oder Fehler kaschieren. Doch der Aufbau eines guten therapeuti-

\section{Die Bewahrung des Arztgeheimnisses hat ihren Preis, und man muss sich dafür engagieren.}

schen Netzwerks setzt voraus, dass Ärztinnen und Ärzte sich gegenseitig unterstützen und aus ihren «Irrtümern» lernen oder, besser gesagt, auf ihren Erfahrungen aufbauen können.

Es ist unbestritten, dass die Versicherer die Möglichkeit haben müssen, bestimmte Kontrollen durchzuführen sowie die Erbringung der Leistung und ihre Angemessenheit zu überprüfen, aber diese Kontrollen dürfen nicht die Gesundheit der Patientinnen und Patienten beeinträchtigen. Die Weitergabe von DRG-Daten sollte vollkommen anonym erfolgen, und es sollte klar zwischen der wirtschaftlichen Prüfung der Leistungen und der Prüfung der Behandlung selbst unterschieden werden; Letztere kann grundsätzlich nur von einer anderen Ärztin oder einem anderen Arzt durchgeführt werden. Die Krankenversicherer sollten sich niemals in die Arzt-Patienten-Beziehung einmischen.

Schlimmer noch: Das Bundesgesetz über die Invalidenversicherung [4] sieht vor, dass der behandelnde Arzt dem Arzt der Invalidenversicherung Informationen über seinen Patienten auch gegen dessen Willen weitergeben «kann»: «Gibt die versicherte Person diese Ermächtigung nicht, so kann ein Arzt des regionalen ärztlichen Dienstes die erforderlichen Auskünfte bei den behandelnden Ärzten der versicherten Person einholen. Diese sind von ihrer Schweigepflicht entbunden». Die blosse - nicht belegte - Hoffnung auf Einsparungen hat den Gesetzgeber dazu verleitet, das Arztgeheimnis zu opfern.

Für eine Verbesserung des allgemeinen Gesundheitsniveaus der Bevölkerung sind weder die Innovationen in der Spitzenmedizin noch eine pedantische Kostenkontrolle ausschlaggebend; entscheidend ist vielmehr die Wahrung ethischer Grundsätze, die bereits im Eid des Hippokrates verfochten werden.

Monique Gauthey, Fachärztin, Mitglied des Zentralvorstandes der FMH, Verantwortliche Ressort Spitalärztinnen und -ärzte

\section{Literatur}

1 Woolf SH, Aron L. U.S. Health in International Perspective: Shorter Lives, Poorer Health. The National academies press; 2013. www.nap.edu/catalog.php?record_id=13497

2 Kiefer B. La faillite sanitaire des Etats-Unis. Rev Méd Suisse. 2013;9:440.

3 Europäisches Recht: Richtlinien 95/46/EG und 2002/58/EG, Verordnung (EG) Nr. 45/2001; www.edps.europa.eu

4 Artikel 3c Abs. 4 IVG. 